\title{
Nonperturbative effects in the Standard Model with gauged 1-form symmetry
}

\author{
Mohamed M. Anber ${ }^{a}$ and Erich Poppitz ${ }^{b}$ \\ ${ }^{a}$ Centre for Particle Theory, Department of Mathematical Sciences, Durham University, \\ South Road, Durham DH1 3LE, U.K. \\ ${ }^{b}$ Department of Physics, University of Toronto, \\ 60 St George St., Toronto, ON M5S 1A\%, Canada \\ E-mail: mohamed.anber@durham.ac.uk, poppitz@physics.utoronto.ca
}

ABSTRACT: We study the Standard Model with gauged $\mathbb{Z}_{N=2,3,6}^{(1)}$ subgroups of its $\mathbb{Z}_{6}^{(1)} 1$-form global symmetry, making the gauge group $\frac{\mathrm{SU}(3) \times \mathrm{SU}(2) \times \mathrm{U}(1)}{\mathbb{Z}_{N}}$. We show that, on a finite $\mathbb{T}^{3}$, there are self-dual instantons of fractional topological charge. They mediate baryon- and lepton-number violating processes. We compare their amplitudes to the ones due to the usual BPST-instantons. We find that the small hypercharge coupling suppresses the fractionalinstanton contribution, unless the torus size is taken sub-Planckian, or extra matter is added above the weak scale. We also discuss these results in light of the cosmological bounds on the torus size.

Keywords: Anomalies in Field and String Theories, Discrete Symmetries, Gauge Symmetry, Nonperturbative Effects

ArXiv EPrint: 2110.02981 


\section{Contents}

1 Introduction, summary, and outlook 1

2 The $\mathbb{Z}_{6}^{(1)}$ and its gauging via twisted boundary conditions 2

2.1 The $\mathbb{T}^{4}$ boundary conditions: transition functions and gauge transformations 3

$2.2 G_{\mathrm{SM}}^{1}$ and the $\mathbb{Z}_{6}^{(1)}$ global symmetry 5

2.3 The gauging of $\mathbb{Z}_{N}^{(1)} \in \mathbb{Z}_{6}^{(1)}$ and $G_{\mathrm{SM}}^{N=2,3,6}$

3 The fractional instantons of $G_{\mathrm{SM}}^{N}$, their actions, and the $\Delta B \neq 0$ amplitudes

3.1 General remarks and concerns 9

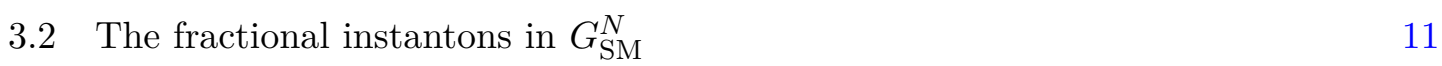

$\begin{array}{ll}3.3 \text { Comparing the } \Delta B \neq 0 \text { rates } & 13\end{array}$

4 Cosmological bounds and discussion $\quad 16$

\section{Introduction, summary, and outlook}

It is well established that the Lie algebra of the Standard Model (SM) is $g_{\mathrm{SM}}=s u(3) \times$ $s u(2) \times u(1)$. The fact that there is freedom to choose different gauge groups $G_{\mathrm{SM}}$ is less broadly appreciated $[1,2]$. If the gauge group is chosen to be $G_{\mathrm{SM}}^{1} \equiv \mathrm{SU}(3) \times \mathrm{SU}(2) \times \mathrm{U}(1)$, the SM with its known matter content has a 1 -form ${ }^{1} \mathbb{Z}_{6}^{(1)}$ global symmetry. The global structure of the SM gauge group can be changed upon gauging its various discrete subgroups, leading to possible gauge groups $G_{\mathrm{SM}}^{N}=\frac{\mathrm{SU}(3) \times \mathrm{SU}(2) \times \mathrm{U}(1)}{\mathbb{Z}_{N}}$, with $N=1,2,3,6$. The global structure of the SM was discussed in [2], using the language of "genuine line operators" of [1]. Refs. [6-11] studied gauge-anomaly related aspects of $G_{\mathrm{SM}}^{N}$, in particular showing the absence of global anomalies in the SM with $G_{\mathrm{SM}}^{N}$.

There are various ways to exhibit the $\mathbb{Z}_{6}^{(1)} 1$-form symmetry of the SM. In this paper, we take an "old-fashioned" approach, initiated by 't Hooft $[12,13]$ in the study of confinement in Yang-Mills theory (see also [14-16] and the recent work [17, 18]). Our main goal is to begin a discussion of the dynamical effects due to the different choices of $G_{\mathrm{SM}}^{N}$. To this end, we consider the SM in a finite volume, taking space to be a three-torus $\mathbb{T}^{3}$. As we review below, the choice of SM gauge group $G_{\mathrm{SM}}^{N}$ is reflected in the boundary conditions on the gauge and matter fields imposed on the spatial $\mathbb{T}^{3}$, in the boundary conditions in the time direction, and in the way the sum over the sectors with different boundary conditions is performed in the path integral.

\footnotetext{
${ }^{1}$ The superscript $G^{(q)}$, with $q=1$, is used to distinguish 1-form symmetries (with group $G$ ), acting on line operators, from ordinary 0-form symmetries $(q=0)$ acting on local operators. The modern introduction of higher-form symmetries is found in [3]. The recent interest in the global aspects of gauge theories and their higher-form symmetries arose due to the need to resolve puzzles related to the mapping of Wilson and 't Hooft line operators under $S$-duality of $\mathcal{N}=4$ super-Yang-Mills [1]. Further interest is due to the importance of higher-form symmetries for the recently discovered [3-5] generalized 't Hooft anomalies.

${ }^{2}$ We remind the reader that "cosmic topology" is a subject with a long history, reviewed in [19].
} 
Our main point, using insight due to 't Hooft [20], is to show that for $G_{\mathrm{SM}}^{N}$, with $N \neq 1$, on a finite $\mathbb{T}^{3}$ there exist self-dual instanton solutions of fractional topological charges. They mediate processes where the baryon number changes by a nonzero amount $\Delta B$, with integer $\Delta B=\Delta L$ (see table 1 below). We calculate the exponential suppression factor for such processes for the various choices of SM gauge group $G_{\mathrm{SM}}^{N}$ and compare it with that for similar processes mediated by the usual BPST [21] instantons computed by 't Hooft [22, 23]. Owing to the fact that these fractional instantons are much less understood ${ }^{3}$ than the BPST instantons, we use the constant field strength self-dual solutions of $[20,26]$. We find that due to the smallness of the hypercharge coupling, the fractional-instanton mediated processes are exponentially suppressed compared to the usual BPST instantons. This suppression can be overcome if the torus size is taken extremely small (sub-Planckian, with only SM content) or if extra matter is added to make the hypercharge coupling increase faster above the weak scale. We also discuss our findings in light of the cosmological bounds on the $\mathbb{T}^{3}$ size $^{4}$ and speculate on the possible cosmological relevance of the global choice $G_{\mathrm{SM}}^{N}$.

We leave various interesting aspects for future work. The way the solutions of [20] are modified in the broken phase of the SM has not yet been studied. It would also be of interest to study the generalization and extension of the construction of non-constant field strength fractional instantons of $[24,25]$ to the SM case. A better control of these issues might help clarify the possible role of the fractional instantons at larger torus sizes.

\section{The $\mathbb{Z}_{6}^{(1)}$ and its gauging via twisted boundary conditions}

In this section, we explain how the $\mathbb{Z}_{6}^{(1)} 1$-form symmetry of the SM naturally arises upon formulating the theory on a spatial 3-torus. This formulation is a convenient starting point to study the fractional topological charge solutions. We also discuss the gauging of the symmetry and its relation to the boundary conditions imposed on the gauge fields in the path integral. (An equivalent description of the gauging of the 1-form symmetry takes the form of a coupling to a 2-form topological quantum field theory [30] and also requires that the spacetime manifold has noncontractible 2-cycles.)

To begin, we denote the $\mathrm{SU}(3) \times \mathrm{SU}(2) \times \mathrm{U}(1)$ gauge couplings $g_{3}, g_{2}$, and $g_{1}$, respectively. For use below, note that the relation between the U(1) coupling and the usual hypercharge coupling $g_{Y}$ is $g_{1}=\frac{g_{Y}}{6}$, because we take all U(1) charges to be integer, due to the assumed compactness of the U(1) gauge group. Explicitly, the charge assignments of the various SM matter fields are standard, except that the hypercharge is multiplied by 6 :

\begin{tabular}{c|ccc|cc} 
field & $\mathrm{SU}(3)$ & $\mathrm{SU}(2)$ & $\mathrm{U}(1)$ & $\mathrm{U}(1)_{B}$ & $\mathrm{U}(1)_{L}$ \\
\hline$q_{L}$ & $\square$ & $\square$ & 1 & $\frac{1}{3}$ & 0 \\
$l_{L}$ & $\mathbf{1}$ & $\square$ & -3 & 0 & 1 \\
$\tilde{e}_{R}$ & $\mathbf{1}$ & $\mathbf{1}$ & 6 & 0 & -1 \\
$\tilde{u}_{R}$ & $\square$ & $\mathbf{1}$ & -4 & $-\frac{1}{3}$ & 0 \\
$\tilde{d}_{R}$ & $\square$ & $\mathbf{1}$ & 2 & $-\frac{1}{3}$ & 0 \\
$h$ & $\mathbf{1}$ & $\square$ & 3 & 0 & 0
\end{tabular}.

\footnotetext{
${ }^{3}$ See $[24,25]$ for relevant recent work.

${ }^{4}$ See [19] and, e.g. [27-29] for more recent work.
} 
All fields above (but the Higgs $h$ ) are left-handed Weyl spinors and we have not included a right-handed neutrino as its possible existence will play no role in our discussion. For use below, in the two r.h. columns we also give the standard baryon and lepton number assignments. In (2.1), we only show a single generation of fermions.

When formulated on a manifold of nontrivial topology, a gauge theory is defined on coordinate patches covering the manifold, and the gauge fields on different patches are related by transition functions, which are gauge group elements. The matter fields on different patches are also related by the transition functions taken in the relevant representation of the gauge group. Here, following [12-15], we consider the case of a torus, which can be described by only one patch, but with nontrivial boundary conditions on the fields. If we were only interested in a Hamiltonian formulation of the theory, we would consider a spatial three-torus $\mathbb{T}^{3}$. In a path integral formulation, we study the finite temperature partition function of the theory on a four-torus $\mathbb{T}^{4} \simeq \mathbb{T}^{3} \times S^{1}$, where $\mathbb{S}^{1}$ is interpreted as a thermal circle; if we wish to approach the zero-temperature limit, we take the size of the circle to infinity.

\subsection{The $\mathbb{T}^{4}$ boundary conditions: transition functions and gauge transformations}

We shall now discuss how the $Z_{6}^{(1)}$ symmetry arises on $\mathbb{T}^{4}$. The $\mathbb{T}^{4}$ is covered by a single closed coordinate patch $0 \leq x^{\mu} \leq a^{\mu}, \mu=1,2,3,4$. This single-patch construction can be seen as a limit of a multiple patch covering of the $\mathbb{T}^{4}$, as is explicitly discussed in [14]. The gauge fields on the different sides of the $\mathbb{T}^{4}$, e.g. at $x^{\mu}=0$ and $x^{\mu}=a^{\mu}$ are related by transition functions, gauge group elements $\Omega_{\mu}$, which can be thought of as defined on the $x^{\mu}=a^{\mu}$ sides of the $\mathbb{T}^{4}$; gauge transformations are also group elements defined on the entire coordinate patch.

To state the boundary conditions, begin by considering an arbitrary $\mu \nu 2$-plane of the torus of sides $a^{\mu}$ and $a^{\nu}$. The coordinates are identified as $x^{\mu} \equiv x^{\mu}+a^{\mu}$. Thus, the 2-plane is a square $0 \leq x^{\mu} \leq a^{\mu}, 0 \leq x^{\nu} \leq a^{\nu}$ (for fixed values of the other coordinates). The fields on opposite sides of the square are related by transition functions, as we now describe. To set our notation, let the gauge fields of the SM be $A_{(j)}=A_{(j)} \mu d x^{\mu}$, with $j=1,2,3$ denoting the $\mathrm{U}(1), \mathrm{SU}(2)$, and $\mathrm{SU}(3)$ gauge fields, respectively. Here $A_{(j) \mu}=A_{(j) \mu}^{a} T_{(j)}^{a}$, where $T_{(j)}^{a}$ are the corresponding group generators in the fundamental representation, $\operatorname{tr} T_{(j)}^{a} T_{(j)}^{b}=\frac{1}{2} \delta^{a b}$, $j=2,3$. For $\mathrm{U}(1), T_{(1)}^{a}$ are replaced by the identity. The gauge field action for the $j$-th gauge group is

$$
S_{(j)}=\frac{1}{4 g_{(j)}^{2}} \int d^{4} x F_{(j) \mu \nu}^{a} F_{(j)}^{\mu \nu a},
$$

where for $j=1$ there is no sum over $a$. The covariant derivative acting on, e.g., $q_{L}$ with charges from (2.1), is then $D_{\mu} q_{L}=\left(\partial_{\mu}+i A_{(1) \mu}+i A_{(2) \mu}^{a} T_{(2)}^{a}+i A_{(3) \mu}^{a} T_{(3)}^{a}\right) q_{L}$. Gauge transformations of the $j$-th group act on the gauge fields as $A_{(j)} \rightarrow g_{(j)}\left(A_{(j)}-i d\right) g_{(j)}^{-1}$. On the matter fields, these are taken in the appropriate representation, e.g. $q_{L} \rightarrow g_{(j)} q_{L}$, where $g_{(j)}$ is a group element in the fundamental representation defined in the $0 \leq x^{\mu} \leq a^{\mu}$ coordinate patch $(\mu=1, \ldots 4)$. For $\mathrm{U}(1)$, the gauge group element is simply $g_{(1)}=e^{i \omega_{(1)}}$ when acting on a field of the minimal quantized unit charge. 
The boundary conditions on the gauge and matter fields in the $\mu$-th direction of the square are then given by

$$
\begin{aligned}
A_{(j)}\left(x^{\mu}=a^{\mu}\right) & =\Omega_{(j) \mu}\left(A_{(j)}\left(x^{\mu}=0\right)-i d\right) \Omega_{(j) \mu}^{-1}, \\
\psi^{\mathcal{R}}\left(x^{\mu}=a^{\mu}\right) & =\Omega_{(1) \mu}^{\mathcal{R}} \Omega_{(2) \mu}^{\mathcal{R}} \Omega_{(3) \mu}^{\mathcal{R}} \psi^{\mathcal{R}}\left(x^{\mu}=0\right), \quad \text { where } \psi^{\mathcal{R}}=\left(q_{L}, l_{L}, \tilde{e}_{R}, \tilde{u}_{R}, \tilde{d}_{R}, h\right) .
\end{aligned}
$$

Above, we did not explicitly indicate the fact that the fields depend on all but the $x^{\mu}$ coordinates, i.e. their values are taken on the 3 -planes $x^{\mu}=a^{\mu}$ or $x^{\mu}=0$ in $\mathbb{T}^{4}$. The boundary conditions for the $\nu$-th direction are identical, with $\mu \rightarrow \nu$, and are given in terms of their own transition functions $\Omega_{(j) \nu}$.

Let us now make some comments regarding the boundary conditions (2.3):

1. The notation in (2.3) may be somewhat condensed, so let us elaborate. The functions $\Omega_{(j) \mu}$ are the transition functions in the $\mu$-th direction of the torus for the $j$-th gauge group. They are group elements of the $j$-th gauge group in the fundamental representation. Note that the transition functions $\Omega_{(j) \mu}$ in the $\mu$-th direction are independent of $x^{\mu}$ and can be thought to be defined at the $x^{\mu}=a^{\mu}$ side of the torus. The matter fields at $x^{\mu}=a^{\mu}$ and $x^{\mu}=0$ are related as shown in the second line in (2.3), where $\Omega_{(j) \mu}^{\mathcal{R}}$ are the transition functions taken in the appropriate representation from (2.1): $\Omega_{(j) \mu}^{\mathcal{R}}$ are the same group elements $\Omega_{(j) \mu}$, but taken in the representation $\mathcal{R}$ of the fermion $\psi^{\mathcal{R}}$. Explicitly, from eq. (2.1), we see that for the SM and for $j=2,3$ only the fundamental and antifundamental representations appear, hence $\Omega_{(j) \mu}^{\mathcal{R}}=\Omega_{(j) \mu}$ if the field $\psi^{\mathcal{R}}$ is in the fundamental representation or $\Omega_{(j) \mu}^{\mathcal{R}}=\Omega_{(j) \mu}^{*}$ if it is in the antifundamental representation. For a $\psi^{\mathcal{R}}$ field of charge $q_{1}$ under $\mathrm{U}(1)$, we have $\Omega_{(1) \mu}^{\mathcal{R}=q_{1}}=\left(\Omega_{(1) \mu}\right)^{q_{1}}$, where $\Omega_{(1) \mu}=e^{i \omega_{(1) \mu}}$ is a compact-U(1) group element. It is clear that with this definition, the matter field and its covariant derivative obey the boundary conditions (2.3).

2. Consistency of the boundary conditions (2.3) with gauge transformations of each gauge group requires that the transition functions $\Omega_{(j) \mu}$ themselves transform under gauge transformations: $\Omega_{(j) \mu} \rightarrow g_{(j)}\left(x^{\mu}=a^{\mu}\right) \Omega_{(j) \mu} g_{(j)}^{-1}\left(x^{\mu}=0\right)$, and similar for the matter fields, where $\Omega$ and $g$ are to be taken in the appropriate representation. For example, when studying classical solutions, one can make use of this freedom to choose a convenient form of the transition functions. Using this gauge freedom to, e.g., set some transition functions to unity, amounts to partial gauge fixing (see below).

3. Most important for our further discussion is the fact that the transition functions obey consistency conditions in every $\mu \nu$-plane of the torus. These are a consequence of the well-known "cocycle conditions" on general manifolds and we shall also call them by that name. In our single-chart setup the cocycle conditions follow by requiring that the fields are single valued on every $\mu \nu$-square. To make the point, consider the matter field $\psi^{\mathcal{R}}$. The value of $\psi^{\mathcal{R}}$ at the $\left(x^{\mu}=a^{\mu}, x^{\nu}=a^{\nu}\right)$ corner of the square can be obtained from its value at the opposite $\left(x^{\mu}=x^{\nu}=0\right)$ corner by using the boundary conditions (2.3) in two different ways: first go along the $x^{\mu}=0$ side in the 
$\nu$-th direction and then along the $x^{\nu}=a^{\nu}$ side in the $\mu$-th direction, or v.v. Obtaining the same value of the $\psi^{\mathcal{R}}$ field at the corner then requires that

$$
\begin{aligned}
& \Omega_{(1) \mu}^{\mathcal{R}} \Omega_{(2) \mu}^{\mathcal{R}} \Omega_{(3) \mu}^{\mathcal{R}}\left(x^{\nu}=a^{\nu}\right) \times \Omega_{(1) \nu}^{\mathcal{R}} \Omega_{(2) \nu}^{\mathcal{R}} \Omega_{(3) \nu}^{\mathcal{R}}\left(x^{\mu}=0\right) \\
= & \Omega_{(1) \nu}^{\mathcal{R}} \Omega_{(2) \nu}^{\mathcal{R}} \Omega_{(3) \nu}^{\mathcal{R}}\left(x^{\mu}=a^{\mu}\right) \times \Omega_{(1) \mu}^{\mathcal{R}} \Omega_{(2) \mu}^{\mathcal{R}} \Omega_{(3) \mu}^{\mathcal{R}}\left(x^{\nu}=0\right) .
\end{aligned}
$$

This should hold for all $\mu, \nu$ planes and all $\psi^{\mathcal{R}}$ from (2.1).

4. There are consistency conditions similar to (2.4) for the $\Omega_{(j) \mu}$ appearing in the gaugefield boundary conditions. It turns out that there is some freedom in the gauge field cocycle conditions that we describe below. In fact, the different $G_{\mathrm{SM}}^{N}$ theories are distinguished by the conditions obeyed by the transition functions for the gauge fields as we now discuss.

\section{$2.2 \quad G_{\mathrm{SM}}^{1}$ and the $\mathbb{Z}_{6}^{(1)}$ global symmetry}

Here, we discuss the cocycle conditions on the transitions functions for the gauge field and introduce the $\mathbb{Z}_{6}^{(1)}$ symmetry of the SM.

Using the same logic as for the field $\psi^{\mathcal{R}}$ to obtain (2.4), we see that to obtain a single-valued gauge field, it is sufficient ${ }^{5}$ to take the gauge field transition functions to obey a condition identical to $(2.4)$ for each $j=1,2,3$. We call this cocycle condition the $G_{\mathrm{SM}}^{1}$ condition:

$$
G_{\mathrm{SM}}^{1}: \quad \Omega_{(j) \mu}\left(x^{\nu}=a^{\nu}\right) \Omega_{(j) \nu}\left(x^{\mu}=0\right)=\Omega_{(j) \nu}\left(x^{\mu}=a^{\mu}\right) \Omega_{(j) \mu}\left(x^{\nu}=0\right), j=1,2,3 .
$$

The fields summed over in the path integral for $G_{\text {SM }}^{1}$ satisfy the boundary conditions (2.3) with transition functions obeying the cocycle conditions (2.4), (2.5). Of particular interest to us is the fact that the topological charge for $G_{\mathrm{SM}}^{1}$ is integer [12-14], to be discussed below. ${ }^{6}$

Now we can explicitly exhibit the 1 -form $\mathbb{Z}_{6}^{(1)}$ center symmetry of the SM. This is a $\mathbb{Z}_{6}$ global symmetry, parameterized by two integers, $k_{\mu}^{2} \in \mathbb{Z}(\bmod 2)$ and $k_{\mu}^{3} \in \mathbb{Z}(\bmod 3)$, for every spacetime direction $\mu$. In this formulation, the 1 -form center symmetry does not act on any fields, but acts only on the transition functions as follows

$$
\begin{aligned}
Z_{6}^{(1)}: & \Omega_{(1) \mu} \rightarrow e^{i 2 \pi\left(-\frac{k_{\mu}^{2}}{2}-\frac{k_{\mu}^{3}}{3}\right)} \Omega_{(1) \mu}, k_{\mu}^{2} \in\{1,2\}, k_{\mu}^{3} \in\{1,2,3\}, \mu=1,2,3,4, \\
& \Omega_{(2) \mu} \rightarrow e^{i 2 \pi \frac{k_{\mu}^{2}}{2}} \Omega_{(2) \mu} . \\
& \Omega_{(3) \mu} \rightarrow e^{i 2 \pi \frac{k_{\mu}^{3}}{3}} \Omega_{(3) \mu} .
\end{aligned}
$$

\footnotetext{
${ }^{5}$ But not necessary: running ahead, we immediately notice that because the gauge field boundary condition in (2.3) contains both $\Omega$ and $\Omega^{-1}$, one can allow a phase factor equal to a center element of each gauge group to appear on the r.h.s. of (2.5).

${ }^{6}$ It can be shown that, using the gauge freedom discussed in section 2.1 , one can take the $j=2,3$ transition functions obeying (2.5) to be trivial in the spatial $\mathbb{T}^{3}$ directions, $\Omega_{(j)} \mu=1$ for $\mu=1,2,3$, and only have a nontrivial $x^{4}$-direction transition function $\Omega_{(j)} \neq 1$. In this gauge, the transition function in the "time" direction carries all the information about the integer topological charge.
} 
Clearly the action (2.6) is consistent with $\Omega_{(j) \mu}$ being (special unitary, for $j=2,3$ ) group elements.

That the above $\mathbb{Z}_{6}^{(1)}$ is a symmetry follows from the fact that both the boundary conditions (2.3) and the gauge and matter fields' cocycle conditions (2.4), (2.5) are invariant under (2.6). The gauge field boundary condition, the first line in (2.3), is clearly invariant since both $\Omega_{(j) \mu}$ and $\Omega_{(j) \mu}^{-1}$ appear on the r.h.s. The cocycle conditions for the gauge field (2.5) are also obviously invariant, as $\Omega_{(j) \mu}$ appears on both sides of the equations. The matter field boundary conditions from the second line in (2.3) are also invariant, for all $\psi^{\mathcal{R}}$ from (2.1), as is easily checked explicitly and follows from the charge assignments. For example, for $q_{L}$ this follows immediately form the fact that the phase factors on the r.h.s. of (2.6) simply cancel out. That the cocycle conditions for the matter fields (2.4) are also invariant follows from the invariance of the boundary conditions $(2.3)$ for $\psi^{\mathcal{R}}$, as the products $\Omega_{(1) \mu}^{\mathcal{R}} \Omega_{(2) \mu}^{\mathcal{R}} \Omega_{(3) \mu}^{\mathcal{R}}$ are invariant under (2.6) for all $\mathcal{R}$.

We have not yet explained why the symmetry (2.6) is a 1-form symmetry, in the modern terminology of [3]: this is because the only gauge invariant operators that (2.6) acts on are Wilson lines winding around the noncontractible loops of the torus. It is clear that all local gauge invariant operators are blind to a symmetry like (2.6) that only acts on transition functions (since a local operator can always be written using fields within a given coordinate patch). On the other hand, a line operator, such as the fundamental representation Wilson loop of the $j$-th gauge group, winding once around the $x^{\mu}$ direction, has the form

$$
W_{(j) \mu}=\operatorname{tr}\left(\mathcal{P} e^{i \int_{x^{\mu}=0}^{x^{\mu}=a^{\mu}} A_{(j)}} \Omega_{(j) \mu}\right)
$$

where $\mathcal{P}$ denotes path ordering and the transition function is inserted in the trace in order to ensure the invariance of $W_{(j) \mu}$ under gauge transformations $g_{(j)}$ on the $\mathbb{T}^{4}$ (recall the gauge transformation law of $\Omega_{(j) \mu}$ mentioned above). It is now clear that under (2.6), we have that

$$
\begin{aligned}
& W_{(3) \mu} \rightarrow e^{i 2 \pi \frac{k_{\mu}^{3}}{3}} W_{(3) \mu}, \\
& W_{(2) \mu} \rightarrow e^{i 2 \pi \frac{k_{\mu}^{2}}{2}} W_{(2) \mu}, \\
& W_{(1) \mu} \rightarrow e^{i 2 \pi\left(-\frac{k_{\mu}^{2}}{2}-\frac{k_{\mu}^{3}}{3}\right)} W_{(1) \mu},
\end{aligned}
$$

showing that $\mathbb{Z}_{6}^{(1)}$ acts on the above fundamental Wilson loops (thus, they are genuine line operators in the SM, as discussed in great detail in [2]). ${ }^{7}$

\footnotetext{
${ }^{7}$ We also note that the introduction of the 1-form symmetry via its action on transition functions is closest to the way the global $\mathbb{Z}_{N}$ center-symmetry is introduced in $\mathrm{SU}(N)$ lattice gauge theory, as described in [31].
} 


\subsection{The gauging of $\mathbb{Z}_{N}^{(1)} \in \mathbb{Z}_{6}^{(1)}$ and $G_{\mathrm{SM}}^{N=2,3,6}$}

Having explained the way the global $\mathbb{Z}_{6}^{(1)} 1$-form symmetry appears in the SM in this language, we can now introduce the different $G_{\mathrm{SM}}^{N}$ theories obtained upon the gauging of the $\mathbb{Z}_{N=2,3,6}^{(1)}$ subgroups of $\mathbb{Z}_{6}^{(1)}$. We describe the gauging using the $\mathbb{T}^{4}$ formulation of the gauge theory, as the fractional instantons whose role we want to study are only explicitly known for $\mathbb{T}^{4}$.

When the gauge theory is formulated on $\mathbb{T}^{4}$ in the Euclidean path integral formalism, the integral is performed over all gauge fields and transition functions obeying the cocycle conditions (2.4) and (2.5), modulo gauge transformations. The cocycle conditions on the gauge field transition functions in (2.5) are the ones appropriate to the $G_{\mathrm{SM}}^{1}=\mathrm{SU}(3) \times$ $\mathrm{SU}(2) \times \mathrm{U}(1)$ gauge group. As already mentioned, with these cocycle conditions, the topological charge is an integer (see (2.14) and (3.11) below).

Now we come to the gauging of the 1-form symmetry. Adapting the discussion of [30] to our case, the gauging of subgroups of $\mathbb{Z}_{6}^{(1)}$ involves two steps: a modification of the gauge fields' cocycle conditions (2.5) by appropriate center elements and a summation in the path integral over gauge fields obeying all such modified cocycle conditions (possibly including $\mathbb{Z}_{N}$ phase factors called "discrete theta angles," introduced in [1] and described for $G_{\mathrm{SM}}^{N}$ in [2]).

We begin with modified gauge-field cocycle conditions (2.5) for the SM case:

$$
\begin{aligned}
& \Omega_{(3) \mu}\left(x^{\nu}=a^{\nu}\right) \Omega_{(3) \nu}\left(x^{\mu}=0\right)=e^{i 2 \pi \frac{n_{\mu \nu}^{(3)}}{3}} \Omega_{(3) \nu}\left(x^{\mu}=a^{\mu}\right) \Omega_{(3) \mu}\left(x^{\nu}=0\right), \\
& \Omega_{(2) \mu}\left(x^{\nu}=a^{\nu}\right) \Omega_{(2) \nu}\left(x^{\mu}=0\right)=e^{i 2 \pi \frac{n_{\mu \nu}^{(2)}}{2}} \Omega_{(2) \nu}\left(x^{\mu}=a^{\mu}\right) \Omega_{(2) \mu}\left(x^{\nu}=0\right), \\
& \Omega_{(1) \mu}\left(x^{\nu}=a^{\nu}\right) \Omega_{(1) \nu}\left(x^{\mu}=0\right)=e^{i 2 \pi\left(-\frac{n_{\mu \nu}^{(3)}}{3}-\frac{n_{\mu \nu}^{(2)}}{2}\right)} \Omega_{(1) \nu}\left(x^{\mu}=a^{\mu}\right) \Omega_{(1) \mu}\left(x^{\nu}=0\right) .
\end{aligned}
$$

The $x$-independent integers appearing in the cocycle conditions for $\mathrm{SU}(2)$ transition functions, $n_{\mu \nu}^{(2)}=-n_{\nu \mu}^{(2)}$, are defined $(\bmod 2)$ for every 2 -plane, while the ones for $\mathrm{SU}(3), n_{\mu \nu}^{(3)}=-n_{\nu \mu}^{(3)}$, are similarly defined $(\bmod 3)$. The $n_{\mu \nu}^{(2),(3)}$ are known as 't Hooft twists. In the formalism introduced in [30] (which we shall not elaborate upon) the integers $n_{\mu \nu}^{(2),(3)}$ correspond to the integrals, taken over the various 2-planes of the torus, of the appropriate 2-form discrete gauge fields gauging the 1 -form $\mathbb{Z}_{N}^{(1)}$ symmetry of the $\mathrm{SU}(N)$ theory (here $N=2,3$ ). The summation over field configurations obeying (2.11) with $n_{\mu \nu}^{(2)}=\{1,2\}$ and $n_{\mu \nu}^{(3)}=\{1,2,3\}$ amounts to performing the path integral over this topological field. ${ }^{8}$

The twists of the gauge field cocycle conditions in (2.11) are consistent with the cocycle conditions (2.4) of the SM matter fields $\psi^{\mathcal{R}}=\left(q_{L}, l_{L}, \tilde{e}_{R}, \tilde{u}_{R}, \tilde{d}_{R}, h\right)$, as the phase factors

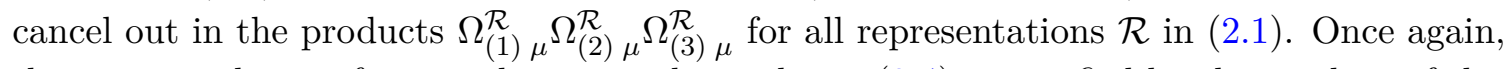
this is most obvious for $q_{L}$, whose cocycle condition (2.4) is satisfied by the product of the three lines in (2.11), and is easily verified for the other SM matter fields.

\footnotetext{
${ }^{8}$ In a Hilbert space interpretation (whose details we shall also not need) this is a summation over discrete magnetic flux sectors and a projection on appropriate discrete electric flux sectors. These sectors were introduced in $[12,13]$. See also $[1,32]$ for somewhat related discussions.
} 
The upshot is that the different $G_{\mathrm{SM}}^{N}$ gauge theories are distinguished by the values of the twists of the cocycle conditions (2.11) obeyed by the transition functions of the gauge and matter fields summed over in the path integral: ${ }^{9}$

$$
\begin{aligned}
& G_{\mathrm{SM}}^{1}=\mathrm{SU}(3) \times \mathrm{SU}(2) \times \mathrm{U}(1): \quad n_{\mu \nu}^{(3)} \equiv 0, \\
& G_{\mathrm{SM}}^{2}=\frac{\mathrm{SU}(3) \times \mathrm{SU}(2) \times \mathrm{U}(1)}{\mathbb{Z}_{2}}: \quad n_{\mu \nu}^{(2)}=\{1,2\}, \quad \text { with } n_{\mu \nu}^{(3)} \equiv 0 \quad \forall \mu, \nu, \\
& G_{\mathrm{SM}}^{3}=\frac{\mathrm{SU}(3) \times \mathrm{SU}(2) \times \mathrm{U}(1)}{\mathbb{Z}_{3}}: \quad n_{\mu \nu}^{(3)}=\{1,2,3\}, \quad \text { with } n_{\mu \nu}^{(2)} \equiv 0 \quad \forall \mu, \nu, \\
& G_{\mathrm{SM}}^{6}=\frac{\mathrm{SU}(3) \times \mathrm{SU}(2) \times \mathrm{U}(1)}{\mathbb{Z}_{6}}: \quad n_{\mu \nu}^{(2)}=\{1,2\}, \quad n_{\mu \nu}^{(3)}=\{1,2,3\} .
\end{aligned}
$$

From our point of view, the most important effect of the gauging of $\mathbb{Z}_{6}^{(1)}$ subgroups in the SM is the fact that the topological charge of gauge fields obeying the modified cocycle conditions (2.5) can be fractional. It was first argued by 't Hooft in [12, 13], and rigorously shown in [14], that the fractional part of the topological charge is determined by the twists $n_{\mu \nu}^{(2),(3)}$. In particular, the topological charge for the $j$-th gauge group is (recalling $F_{(j)}=\frac{1}{2} F_{(j) \mu \nu} d x^{\mu} \wedge d x^{\nu}$ and noting that there is no trace for $\left.j=1\right)$ :

$$
Q_{(j)}=\frac{1}{8 \pi^{2}} \int \operatorname{tr} F_{(j)} \wedge F_{(j)}= \begin{cases}\frac{1}{32 \pi^{2}} \int d^{4} x F_{(j)}^{\mu \nu a} \tilde{F}_{(j) \mu \nu}^{a}, & j=2,3 \\ \frac{1}{16 \pi^{2}} \int d^{4} x F_{(1)}^{\mu \nu} \tilde{F}_{(1) \mu \nu}, & j=1\end{cases}
$$

where $\tilde{F}_{(j) \mu \nu} \equiv \frac{1}{2} \epsilon_{\mu \nu \lambda \sigma} F_{(j)}^{\lambda \sigma}$. For $j=2$ and $j=3$, it was shown in [12-14] that $\mathrm{SU}(j)$ field configurations obeying the cocycle conditions (2.11) have fractional topological charge

$$
Q_{(j)}=-\frac{\operatorname{Pf}\left(n^{(j)}\right)}{j}+k, j=2,3, \text { and } k \in \mathbb{Z} .
$$

Here, $\operatorname{Pf}(n) \equiv \frac{1}{8} \epsilon^{\mu \nu \lambda \sigma} n_{\mu \nu} n_{\lambda \sigma}$ is an integer for an antisymmetric matrix with integer entries. The simplest example is to take $n_{12}=-n_{21}=n_{34}=-n_{34}=1$ as the only nonzero entries of $n_{\mu \nu}$, giving $\operatorname{Pf}(n)=n_{12} n_{34}=1$.

We used eq. (2.13) to define the quantity $Q_{(1)}$ characterizing the $\mathrm{U}(1)$ gauge field configuration, noting that there is no $\mathrm{U}(1)$ characteristic class associated with the $\mathbb{T}^{4}$.

\footnotetext{
${ }^{9}$ To avoid confusion, we stress that one can, even in the $G_{\mathrm{SM}}^{1}$ theory, introduce boundary conditions on the $\mathbb{T}^{4}$ that correspond to nonzero twists $n_{\mu \nu}^{(j)}$, but without summation over all allowed twists in the path integral. This corresponds to probing the $G_{\mathrm{SM}}^{1}$ theory by the insertion of nondynamical backgrounds for the $\mathbb{Z}_{6}^{(1)}$ global symmetry, needed in studies of generalized 't Hooft anomalies involving center symmetry, as shown using this language in [32]. An unambiguous identification of the different SM gauge groups with the presence or absence of fractional instanton contributions requires forbidding such nondynamical insertions. This is natural in the context of embedding the SM in quantum gravity theories, where global symmetries cannot appear; for example, in the case of $G_{\mathrm{SM}}^{(1)}$, the $\mathbb{Z}_{6}^{(1)}$ symmetry is broken by the presence of heavy electric states whose transition functions do not respect $(2.6)$, disallowing nonzero twists in $G_{\mathrm{SM}}^{(1)}$. Likewise, global $\mathbb{Z}_{3}^{(1)}$ twists can be forbidden in $G_{\mathrm{SM}}^{(2)}$ and $\mathbb{Z}_{2}^{(1)}$ ones in $G_{\mathrm{SM}}^{(3)}$. It is also worth mentioning that there is a $\mathbb{Z}_{3}$ magnetic symmetry in $G_{\mathrm{SM}}^{(3)}$ and $G_{\mathrm{SM}}^{(6)}$, which is broken by heavy magnetic charges in theories of quantum gravity. See [2] and references therein for a discussion of these issues in the present context.
} 
Instead, the U(1) background is characterized by the fluxes through noncontractible 2planes ("first Chern class"). With nontwisted cocycle conditions for the U(1), i.e. with $\mathrm{U}(1)$ transition functions obeying (2.5), field configurations are characterized by $2 \pi \times$ integer fluxes through the various 2 -planes, e.g. $\oint d x^{1} d x^{2} F_{(1) 12}=2 \pi \mathbb{Z}$. It is easy to see that our $Q_{(1)}$ of (2.14) then takes integer values (see eq. (3.11) below). For U(1) field configurations obeying the cocycle conditions from the last line in (2.11), which are modified by the $\mathbb{Z}_{6}$ twists $\bar{n}_{\mu \nu}$ (with $\left.\frac{\bar{n}_{\mu \nu}}{6} \equiv \frac{-2 n_{\mu \nu}^{(3)}-3 n_{\mu \nu}^{(2)}}{6}\right)$, these fluxes through 2-planes are now valued in $\frac{2 \pi \mathbb{Z}}{6}$ and $Q_{(1)}$ is further reduced, as also seen in (3.11) below.

The fact that the topological charge is fractional leads one to expect that the physical effects of such topological fluctuations may be less suppressed than the ones due to the well-understood integer topological charge BPST instantons. The gauge field Euclidean action (2.2) can be rewritten in terms of the topological charge $Q_{(j)}($ for $j=2,3)$ as

$$
S_{(j)}= \pm \frac{8 \pi^{2}}{g_{(j)}^{2}} Q_{(j)}+\frac{1}{8 g_{(j)}^{2}} \int d^{4} x\left(F_{(j) \mu \nu} \mp \tilde{F}_{(j) \mu \nu}\right)^{2}
$$

where the signs are correlated, showing that the action for field configurations with $Q_{(j)}>0$ are minimized by self-dual fields $F_{(j) \mu \nu}=\tilde{F}_{(j) \mu \nu}$ (and anti self-dual ones for negative topological charge). Thus, the minimal action in each topological sector $Q_{(j)}$ (for $j=2,3$ ) is given by the familiar Bogomolny' bound

$$
S_{\min }=\frac{8 \pi^{2}}{g_{(j)}^{2}}\left|Q_{(j)}\right|=\frac{8 \pi^{2}}{g_{(j)}^{2}}\left|\frac{\operatorname{Pf}\left(n^{(j)}\right)}{j}-k\right| .
$$

For example, with $\left|Q_{(j)}\right|=\frac{1}{j}$, the action is smaller than that of a BPST instanton. In particular, solutions whose action scales as $8 \pi^{2} /\left(g^{2} N\right)$, as above, with $j \rightarrow N$, give contributions to the path integral which survive in the large- $N$ limit - which was the initial motivation of their study in [20].

Before we continue, we recall the anomaly equation for the $\mathrm{U}(1)_{B}$ baryon number of (2.1),

$$
\partial_{\mu} j_{B}^{\mu}=\frac{1}{32 \pi^{2}} F_{(2)}^{\mu \nu a} \tilde{F}_{(2) \mu \nu}^{a}-18 \frac{1}{16 \pi^{2}} F_{(1)}^{\mu \nu} \tilde{F}_{(1) \mu \nu},
$$

which implies that the change of baryon number is $\Delta B=Q_{(2)}-18 Q_{(1)}$, for a single generation of SM fields. In the next section, we describe the $G_{\mathrm{SM}}^{N}$ fractional instantons and study their effect.

\section{The fractional instantons of $G_{\mathrm{SM}}^{N}$, their actions, and the $\Delta B \neq 0$ amplitudes}

\subsection{General remarks and concerns}

Before we exhibit the fractional instantons for the $G_{\mathrm{SM}}^{N}$ theories that we shall use as our illustrative example, we note that the current knowledge of such solutions is somewhat limited. The fact that the topological charge is fractional has been known since [12-14], but there are only a few explicit solutions. 
Below, as a first step to investigating the possible role of fractional instantons, we make two simplifications, both of which should be reconsidered in future studies. First, we set the vev of the Higgs field to zero assuming a symmetric phase. Second, we use the constant field strength solutions on $\mathbb{T}^{4}$ of [20], and, in particular, their abelian version. These analytic solutions saturate the Bogomolny' bound (2.16) and are stable only for a particular ratio of sides of the $\mathbb{T}^{4}[26]$. In particular, using these solutions, we are forced to take the $\mathbb{T}^{4}$ sides to obey

$$
\frac{a^{3} a^{4}}{a^{1} a^{2}}=1
$$

This constraint ensures that both the $j=2$ and $j=3$ fractional instantons are self-dual and obey the Bogomolny' bound (2.16). The $\mathrm{SU}(3)$ action of these solutions, however, is only $2 / 3$ the BPST instanton action (rather than the minimal ${ }^{10} 1 / 3$ ), while the $\mathrm{SU}(2)$ action is the minimum possible $1 / 2$ of the BPST action.

Clearly, the constraint (3.1) is a source of concern. First, it limits the ability to independently vary the shape and size of the spatial $\mathbb{T}^{3}$ and of the time direction extent $a_{4}$. In particular, it does not incorporate zero-temperature fractional instantons on $\mathbb{R} \times \mathbb{T}^{3}[16]$. Further, since the solutions have field strength uniform over the torus, one expects them to be relevant for possible physical applications only if the size of the torus is smaller than the causally connected part of the universe. As we discuss below, this fact, along with the cosmological bounds on the torus size, would then imply that the SM fractional instantons are mostly of academic interest.

While this may ultimately turn out to be the case, let us make several remarks pointing towards a possible way out. There exist numerical studies as well as an analytic approach towards finding fractional instantons on tori not restricted by (3.1) or its generalization. More precisely, fractional instanton solutions can be constructed by relaxing the ratio $\frac{a^{3} a^{4}}{a^{1} a^{2}}=f(N, k, l)$, with $k+l=N$ ('t Hooft's generalization of (3.1)) towards arbitrary values of $a^{\mu}$, using a perturbative approach, developed in [24, 25]. It is shown there that the fractional instanton solutions thus obtained have a nontrivial profile of the field strength over the torus. The instanton size is, however, still set by the torus dimensions (the equations studied are the scale-free Yang-Mills equations on a torus with twisted boundary conditions, as in the top line of (2.11)). It appears, then, that fractional instantons whose size does not scale with the size of space (time) would require the introduction of an extra scale. This could be related to the strong scale of QCD or to the electroweak scale, ${ }^{11}$ the Higgs vev that we neglect throughout. It is thus of interest to study especially (in the present context) the effect of re-introducing the Higgs vev.

With our concerns spelled out, we now describe the fractional instantons on $\mathbb{T}^{4}$.

\footnotetext{
${ }^{10}$ We note that 't Hooft [20] constructed $\mathrm{SU}\left(N_{c}=3\right)$ nonabelian constant field strength solutions of the minimal possible action (2.16), equal to $1 / 3$ times the BPST action. However, the relation replacing (3.1) and ensuring self-duality then has the ratio $a^{3} a^{4} /\left(a^{1} a^{2}\right)$ replaced with a quantity $f\left(N_{c}, k, l\right)$ (with $k+l=N_{c}$ ) that depends on $N_{c}$. Hence, self duality is not obeyed for both $N_{c}=2$ and $N_{c}=3$, prompting us to use the abelian version. The generalization of these solutions to product-group structures such as the SM has not been studied.

${ }^{11}$ Notice that it is precisely the appearance of an extra scale, due to the holonomy vev, that reveals the fractional instantons in the much better understood case of $\mathbb{R}^{3} \times \mathbb{S}^{1}[33,34]$.
} 


\subsection{The fractional instantons in $G_{\mathrm{SM}}^{N}$}

To describe the fractional instantons explicitly, we introduce $H^{(3)}$ and $H^{(2)}$, diagonal matrices in the Cartan subalgebra of $\mathrm{SU}(3)$ and $\mathrm{SU}(2)$ :

$$
H^{(3)}=\operatorname{diag}\left(\frac{1}{3}, \frac{1}{3},-1+\frac{1}{3}\right), H^{(2)}=\operatorname{diag}\left(\frac{1}{2},-\frac{1}{2}\right), \operatorname{tr} H^{(3)} H^{(3)}=\frac{2}{3}, \operatorname{tr} H^{(2)} H^{(2)}=\frac{1}{2} .
$$

The fractional instantons are the following field configurations, where $n_{\mu \nu}^{(3),(2)}$ are the twists appearing in (2.11), as we show below:

$$
\begin{aligned}
& A_{(j)}=\sum_{\mu, \nu=1}^{4} \frac{\pi x^{\nu} d x^{\mu} n_{\mu \nu}^{(j)}}{a^{\mu} a^{\nu}} H^{(j)}, \text { for } j=2,3, \\
& A_{(1)}=-\sum_{\mu, \nu=1}^{4} \frac{\pi x^{\nu} d x^{\mu}}{a^{\mu} a^{\nu}}\left(\frac{3 n_{\mu \nu}^{(2)}+2 n_{\mu \nu}^{(3)}}{6}+n_{\mu \nu}^{(1)}\right) .
\end{aligned}
$$

In the last line, we introduced an integer-valued $n_{\mu \nu}^{(1)}=-n_{\nu \mu}^{(1)}$ twist reflecting the freedom to add $2 \pi \mathbb{Z}$ fluxes of the U(1) field. Notice that the gauge fields (3.3) are not periodic, but instead obey

$$
\begin{aligned}
& A_{(j)}\left(x^{\lambda}=a^{\lambda}\right)-A_{(j)}\left(x^{\lambda}=0\right)=\sum_{\mu}^{4} \frac{\pi d x^{\mu} n_{\mu \lambda}^{(j)}}{a^{\mu}} H^{(j)} \equiv-i \Omega_{(j) \lambda} d \Omega_{(j) \lambda}^{-1}, \text { for } j=2,3, \\
& A_{(1)}\left(x^{\lambda}=a^{\lambda}\right)-A_{(2)}\left(x^{\lambda}=0\right)=-\sum_{\nu=1}^{4} \frac{\pi d x^{\mu}}{a^{\mu}}\left(\frac{3 n_{\mu \lambda}^{(2)}+2 n_{\mu \lambda}^{(3)}}{6}+n_{\mu \nu}^{(1)}\right) \equiv-i \Omega_{(1) \lambda} d \Omega_{(1) \lambda}^{-1},
\end{aligned}
$$

from which we can read off the transition functions

$$
\Omega_{(j) \lambda}=e^{-i \sum_{\mu} \frac{\pi x^{\mu} n_{\mu \lambda}^{(j)}}{a^{\mu}} H^{(j)}}, \text { for } j=2,3 \text {, while } \Omega_{(1) \lambda}=e^{i \sum_{\mu} \frac{\pi x^{\mu}}{a^{\mu}}\left(\frac{3 n_{\mu \lambda}^{(2)}+2 n_{\mu \lambda}^{(3)}}{6}+n_{\mu \nu}^{(1)}\right)} .
$$

We can now ask what cocycle conditions the above transition functions obey. To this end, we compute the 1.h.s. and r.h.s. of (2.11). For $j=3$ and $j=2$, we have for the 1.h.s. of $(2.11)$ :

$$
\Omega_{(j) \lambda}\left(x^{\rho}=a^{\rho}\right) \Omega_{(j) \rho}\left(x^{\lambda}=0\right)=e^{-i \pi n_{\rho \lambda}^{(j)} H^{(j)}} e^{-i \sum_{\mu \neq \rho} \frac{\pi x^{\mu} n_{\mu \lambda}^{(j)}}{a^{\mu}} H^{(j)}} e^{-i \sum_{\mu \neq \lambda} \frac{\pi x^{\mu} n_{\mu \rho}^{(j)}}{a^{\mu}} H^{(j)}},
$$

while the combination of transition functions (3.5) appearing on the r.h.s. of (2.11) is

$$
\Omega_{(j) \rho}\left(x^{\lambda}=a^{\lambda}\right) \Omega_{(j) \lambda}\left(x^{\rho}=0\right)=e^{-i \pi n_{\lambda \rho}^{(j)} H^{(j)}} e^{-i \sum_{\mu \neq \lambda} \frac{\pi x^{\mu} \mu_{\mu \rho}^{(j)}}{a^{\mu}} H^{(j)}} e^{-i \sum_{\mu \neq \rho} \frac{\pi x^{\mu} n_{\mu \lambda}^{(j)}}{a^{\mu}} H^{(j)}} .
$$

Comparing the two expressions (3.6) and (3.7), using the antisymmetry of $n_{\lambda \rho}^{(j)}$, we find that (3.5) obey the cocycle condition

$$
\Omega_{(j) \lambda}\left(x^{\rho}=a^{\rho}\right) \Omega_{(j) \rho}\left(x^{\lambda}=0\right)=e^{i 2 \pi n_{\lambda \rho}^{(j)} H^{(j)}} \Omega_{(j) \rho}\left(x^{\lambda}=a^{\lambda}\right) \Omega_{(j) \lambda}\left(x^{\rho}=0\right) .
$$


Now, recalling from $(3.2)$ that $H^{(3)}=\operatorname{diag}\left(\frac{1}{3}, \frac{1}{3},-1+\frac{1}{3}\right)$ and $H^{(2)}=\operatorname{diag}\left(\frac{1}{2},-\frac{1}{2}\right)$, we see that (3.8) produces precisely (2.11) for $j=2,3$. It is trivial to check that the remaining $\mathrm{U}(1)$ condition in (2.11) also holds; notice that the $n_{\mu \nu}^{(1)} \in \mathbb{Z}$ factor does not enter the cocycle condition.

The upshot of the above discussion is that we have shown that (3.3) are gauge field backgrounds obeying the appropriate cocycle conditions (2.11) for gauging the $\mathbb{Z}_{6}^{(1)}$ subgroups, as described in (2.12). Having constant field strength and being abelian, they also solve the gauge field equations of motion as well as the scalar $h$ equation of motion with vev set to zero, i.e. assuming the symmetric phase. The stability of (3.3) is a consequence of the self-duality, which we impose below.

The topological charges (2.13) of our fractional instantons (3.3) are now easily computed. In fact, the $Q_{(j)}$ only depend on the transition functions (3.5) but for the constant field strength background we can compute them using the field strength. For $j=2,3$, we have $F_{(j)}=\sum_{\mu \nu} \frac{\pi d x^{\nu} \wedge d x^{\mu} n_{\mu \nu}^{(j)}}{a^{\mu} a^{\nu}} H^{(j)}$, which yields, using (3.2)

$$
Q_{(2)}=\frac{\operatorname{Pf}\left(n^{(2)}\right)}{2}, Q_{(3)}=\frac{2 \operatorname{Pf}\left(n^{(3)}\right)}{3} .
$$

For the $\mathrm{U}(1)$, we find instead

$$
F_{(1)}=-\sum_{\mu \nu} \frac{\pi d x^{\nu} \wedge d x^{\mu}}{a^{\mu} a^{\nu}}\left(\frac{3 n_{\mu \nu}^{(2)}+2 n_{\mu \nu}^{(3)}}{6}+n_{\mu \nu}^{(1)}\right),
$$

yielding

$$
Q_{(1)}=\frac{1}{8 \pi^{2}} \int F_{(1)} \wedge F_{(1)}=\frac{1}{36} \operatorname{Pf}\left(6 n^{(1)}+3 n^{(2)}+2 n^{(3)}\right) .
$$

As promised earlier, the $\mathrm{U}(1)$ topological charge $Q_{(1)}$ is integer for vanishing $n^{(2)}$ and $n^{(3)}$. From (2.17) we can now immediately compute the $\Delta B$ due to our fractional instantons

$$
\Delta B=\frac{\operatorname{Pf}\left(n^{(2)}\right)}{2}-\frac{1}{2} \operatorname{Pf}\left(6 n^{(1)}+3 n^{(2)}+2 n^{(3)}\right) .
$$

It is easy to see that the change of baryon number (3.12) is always integer.

The actions of the fractional instantons (3.3) can also be computed. The smallest actions of the $\mathrm{SU}(2)$ and $\mathrm{SU}(3)$ fractional instantons are obtained for solutions on a $\mathbb{T}^{4}$ obeying (3.1). The Bogomolny' bound (2.16) holds after setting $\operatorname{Pf}\left(n^{(2)}\right)=\operatorname{Pf}\left(n^{(3)}\right)=1$ by, for example, taking the only nonzero entries $n_{12}^{(j)}=n_{34}^{(j)}=-n_{21}^{(j)}=-n_{43}^{(j)}=1$ for $j=2,3$ :

$$
S_{(2)}=\frac{4 \pi^{2} n_{12}^{(2)}}{g_{2}^{2}}, \quad S_{(3)}=\frac{16 \pi^{2} n_{12}^{(3)}}{3 g_{3}^{2}} .
$$

We note that we have kept $n_{12}^{(j)}$ as a book-keeping device: according to our discussion near eq. (2.12), we set $n_{12}^{(2)}=n_{12}^{(3)}=1$ upon gauging the full $\mathbb{Z}_{6}^{(1)}$, we set $n_{12}^{(2)}=1, n_{12}^{(3)}=0$ when gauging $\mathbb{Z}_{2}^{(1)}$, and $n_{12}^{(2)}=0, n_{12}^{(3)}=1$ when gauging $\mathbb{Z}_{3}^{(1)}$. These values for $n^{(j)}, j=2,3$, give rise to the smallest topological charge for the given gauging. The fractional instantons thus obtained are (anti) self-dual and are necessary stable, as was shown in [26]. 


\begin{tabular}{|c|c|c|c|c|c|}
\hline Gauged 1-form center & $n_{12}^{(1)}$ & $n_{34}^{(1)}$ & Smallest $\mathrm{U}(1)$ action & $\Delta B$ & $\mu_{\text {critical }}(\mathrm{GeV})$ \\
\hline $\mathbb{Z}_{6}^{(1)}$ & -1 & -1 & $\frac{\pi^{2}}{9 g_{1}^{2}}$ & 0 & - \\
\hline $\mathbb{Z}_{6}^{(1)}$ & -1 & 0 & $\frac{13 \pi^{2}}{9 g_{1}^{2}}$ & $3 n_{f}$ & $6 \times 10^{34}$ \\
\hline $\mathbb{Z}_{6}^{(1)}$ & 0 & -1 & $\frac{13 \pi^{2}}{9 g_{1}^{2}}$ & $3 n_{f}$ & $6 \times 10^{34}$ \\
\hline $\mathbb{Z}_{3}^{(1)}$ & 0 & 0 & $\frac{4 \pi^{2}}{9 g_{1}^{2}}$ & $-2 n_{f}$ & $2.7 \times 10^{33}$ \\
\hline $\mathbb{Z}_{2}^{(1)}$ & 0 & 0 & $\frac{\pi^{2}}{g_{1}^{2}}$ & $-4 n_{f}$ & $1.5 \times 10^{24}$ \\
\hline
\end{tabular}

Table 1. The smallest $\mathrm{U}(1)$ action $S_{(1)}$ of fractional instantons, obtained by varying the integervalued $\mathrm{U}(1)$ fluxes $n_{\mu \nu}^{(1)}$, for the different choices of the Standard Model gauge group $G_{\mathrm{SM}}^{N}$. In the two rightmost columns, we show the corresponding value of $\Delta B$ for $n_{f}$ fermion generations and the scale $\mu_{\text {critical }}$ (taking $n_{f}=3$ ) above which the fractional instantons begin to dominate over the BPST instanton.

The action of the $\mathrm{U}(1)$ instantons, on the contrary, needs to be directly computed starting from the U(1) gauge field background (3.3). Without loss of generality, we can consider gauge fields in the $1-2$ and $3-4$ planes and take $n_{12}^{(j)}=-n_{21}^{(j)}=n_{34}^{(j)}=-n_{34}^{(j)}=1$ as the only nonzero entries of $n_{\mu \nu}^{(j)}$, for $j=2,3$, which gives:

$$
S_{(1)}=\frac{2 \pi^{2}}{g_{1}^{2}}\left[\left(\frac{n_{12}^{(3)}}{3}+\frac{n_{12}^{(2)}}{2}+n_{12}^{(1)}\right)^{2}+\left(\frac{n_{12}^{(3)}}{3}+\frac{n_{12}^{(2)}}{2}+n_{34}^{(1)}\right)^{2}\right] .
$$

Notice that $\mathrm{U}(1)$ solutions with $n_{12}^{(1)} \neq n_{34}^{(1)}$ are not (anti) self-dual. Yet, no unstable modes are expected in this case since the quantum fluctuations about U(1) classical background are always free.

\subsection{Comparing the $\Delta B \neq 0$ rates}

As explained above, different choices of $n_{12}^{(2)}, n_{12}^{(3)}$ correspond to gauging different subgroups of $\mathbb{Z}_{6}^{(1)}$ (as per (2.12)) and yield different values of $\Delta B$; the latter also depends on the freedom to add integer $\mathrm{U}(1)$ fluxes $n_{12}^{(1)}, n_{34}^{(1)}$.

Thus, in table 1, we show, for every gauged 1-form center symmetry, the values of $n_{12}^{(1)}$ and $n_{34}^{(1)}$ that yield the smallest $\mathrm{U}(1)$ actions and the corresponding baryon number violation for $n_{f}$ SM generations. ${ }^{12}$

Our next task is to compare the vacuum to vacuum amplitude of $\Delta B$ mediated by the fractional-charge instantons with the amplitude of the same quantity due to the weak selfdual BPST instantons. Ideally, one would want to conduct this study at zero temperature on a background manifold $\mathbb{M}^{3} \times \mathbb{R}$ that admits both types of instantons. Unfortunately,

\footnotetext{
${ }^{12}$ Notice that the smallest $U(1)$ action in the gauged $-\mathbb{Z}_{6}^{(1)}$ case does not result in baryon number violation, and therefore, we needed to consider the next to the smallest action obtained by turning on integer-quantized $\mathrm{U}(1)$ fluxes denoted by $n^{(1)}$. The way the scale $\mu_{\text {critical }}$ is defined is explained in the text; its value is given for the case of $n_{f}=3$ generations.
} 
there is no known background that accommodates analytical solutions of both types. To make matter worse, even on $\mathbb{T}^{3} \times \mathbb{R}$, as we discussed above, analytical solutions of fractional instantons are still lacking. With these obstacles, our best option is to conduct our study on the simple manifold $\mathbb{T}^{4}$. Here, we argue that our treatment gives the correct order of magnitude estimate, which should not be greatly altered by the fine details of the solutions. First, the action of the self-dual fractional instanton, in the Bogomolny' bound, depends only on the topological charge and one can proclaim that deforming $\mathbb{T}^{4}$ into $\mathbb{T}^{3} \times \mathbb{R}$ (by making one of the cycles very large) cannot change the action. ${ }^{13}$ Second, although it is true that there is no solution of self-dual BPST instanton on $\mathbb{T}^{4}$ with zero twists [35], adding a twist removes the obstruction to the existence of the solution. ${ }^{14}$ In our case the twists come about naturally, since we need them anyway to obtain the fractional solutions. This is a win-win situation for both the BPST and fractional solutions. Again, one can imagine a process where we take one of the cycles of $\mathbb{T}^{4}$ large enough to obtain BPST solutions on $\mathbb{T}^{3} \times \mathbb{R}$. A third important point is that baryon number violation is governed mainly by anomalies. The latter depend only on the cohomology classes and not on the specific background manifold, and thus, we expect the computations on $\mathbb{T}^{4}$ or $\mathbb{T}^{3} \times \mathbb{R}$ yield the same estimates.

Given all the above caveats and their suggested resolutions, we proceed to our computations. We begin with $\mathbb{T}^{4}$ of a size much smaller than the inverse $\mathrm{TeV}$ scale and, therefore, we consistently set the Higgs vev to zero and use the solutions (3.3) and the corresponding action for the fractional instantons. As we discussed above, finding BPST instanton (approximate) solutions on $\mathbb{T}^{4}$ should also be possible, with a scale modulus cutoff by the torus size. We then declare that the actions of the BPST and fractional instantons will not be greatly modified as we take one of the $\mathbb{T}^{4}$ cycles large enough, while keeping the $\mathbb{T}^{3}$ size below the inverse $\mathrm{TeV}$ scale, such that we approach the zero-temperature limit $\mathbb{T}^{3} \times \mathbb{R}$. In this limit, the small size of $\mathbb{T}^{3}$ will set the energy scale of the problem. We also give the SM fermions periodic boundary conditions along the spatial directions $\mu=1,2,3$. Effectively, the problem is reduced to quantum mechanics, where there are two types of nonperturbative solutions that compete with each other to win the baryon-number-violation race. The action of the solution(s) is the highest of the barriers between the different vacuum components, and the solution with the smallest action will more effectively violate the baryon number. In this regime the schematic form of 't Hooft vertex in the background of fractional instantons is given by (we consider the contribution from one family of quarks and leptons and the field insertions $q_{L}$ etc. are the fermion zero modes)

$$
\mathcal{T}_{\text {fraction }} \sim e^{-\left(S_{(1)}+S_{(2)}+S_{(3)}\right)}\left(q_{L}\right)^{\mathcal{I}_{q_{L}}}\left(l_{L}\right)^{\mathcal{I}_{l}}\left(\tilde{e}_{R}\right)^{\mathcal{I}_{\tilde{e}_{R}}}\left(\tilde{u}_{R}\right)^{\mathcal{I}_{\tilde{u}_{R}}}\left(\tilde{d}_{R}\right)^{\mathcal{I}_{\tilde{d}_{R}}}
$$

\footnotetext{
${ }^{13}$ In the following, we use the SU(3) solutions with topological charge $\frac{2}{3}$ instead of the solutions with the minimum charge $\frac{1}{3}$ described by 't Hooft [20], see footnote 10. We also assume that the U(1) topological charge and action of the constant-field solution (3.10) does not change dramatically during this process, i.e., when we make one of the $\mathbb{T}^{4}$ cycles very large.

${ }^{14}$ See [16] for a discussion. Simply, if one starts with a self-dual BPST instanton on $\mathbb{R}^{4}$, then deforming it to $\mathbb{T}^{4}$ will be obstructed since the latter manifold enjoys a continuous degeneracy in the moduli space. This degeneracy, however, will be lifted when we apply the twist. Yet, from a practical point of view, one can get very close to self-duality even in the absence of twists.
} 
where $\mathcal{I}_{q_{L}}$, etc. are the Dirac indices:

$$
\begin{array}{ll}
\mathcal{I}_{q_{L}}=2 Q_{(3)}+3 Q_{(2)}+6 Q_{(1)}, & \mathcal{I}_{l_{L}}=Q_{(2)}+18 Q_{(1)}, \quad \mathcal{I}_{\tilde{e}_{R}}=36 Q_{(1)}, \\
\mathcal{I}_{\tilde{u}_{R}}=Q_{(3)}+48 Q_{(1)}, & \mathcal{I}_{\tilde{d}_{R}}=Q_{(3)}+12 Q_{(1)} .
\end{array}
$$

The indices are obtained from the index theorem, which gives the number of the Weyl zero modes in the background of the fractional-charge instanton. The indices (3.16), like $\Delta B$ of (3.12), are always integer, for fermions whose transition functions are consistent with the cocycle conditions (2.4), (2.11) (this can also be explicitly checked). Inserting the vertex into the SM partition function and applying a $\mathrm{U}(1)_{B}$ global transformation gives $\Delta B=\frac{1}{3}\left(\mathcal{I}_{q_{L}}-\mathcal{I}_{\tilde{u}_{R}}-\mathcal{I}_{\tilde{d}_{R}}\right)=Q_{(2)}-18 Q_{(1)}$, which is exactly the result from the anomaly equation (2.17). Let us compare (3.15) to 't Hooft vertex from the weak BPST instantons that give the same $\Delta B$ (per family)

$$
\mathcal{T}_{\mathrm{BPST}} \sim e^{-|\Delta B| S_{\mathrm{BPST}}}\left(q_{L}^{3} l_{L}\right)^{|\Delta B|} .
$$

Bearing in mind that the actions are coupling constant dependent, the sum of the actions $S_{(1)}+S_{(2)}+S_{(3)}$ could become smaller than the action of the BPST instantons (times $\Delta B)$, which, in turn, results in a larger amplitude for the fractional-instanton-mediated process. Using (3.13), (3.14), and $S_{\mathrm{BPST}}=\frac{8 \pi^{2}}{g_{2}^{2}}$, the condition $S_{(1)}+S_{(2)}+S_{(3)}<|\Delta B| S_{\mathrm{BPST}}$ can be rewritten as:

$$
\frac{16 \pi^{2} n_{12}^{(3)}}{3 g_{3}^{2}(\mu)}+\frac{2 \pi^{2}}{g_{1}^{2}(\mu)}\left[\left(\frac{n_{12}^{(3)}}{3}+\frac{n_{12}^{(2)}}{2}+n_{12}^{(1)}\right)^{2}+\left(\frac{n_{12}^{(3)}}{3}+\frac{n_{12}^{(2)}}{2}+n_{34}^{(1)}\right)^{2}\right]<\frac{8 \pi^{2}}{g_{2}^{2}(\mu)}\left(|\Delta B|-\frac{n_{12}^{(2)}}{2}\right) .
$$

As above, $n_{12}^{(2)}$ and $n_{12}^{(3)}$ (taken to be either 0 or 1 ) are used as a book-keeping device to track the gauging of $\mathbb{Z}_{6}^{(1)}$ or subgroups of it, while $n_{12}^{(1)}$ and $n_{34}^{(1)}$ are as in table 1.

As we emphasized in the inequality (3.18), the coupling constants $g_{1,2,3}$ depend on some energy scale $\mu$. Above the electroweak scale, the Higgs is in the symmetric phase and the only scale in the problem is the $\mathbb{T}^{3}$ size, which works as an IR cutoff and sets the energy scale $\mu$. To determine the size of $\mathbb{T}^{3}$ that satisfies the inequality (3.18), we run the coupling constants from the weak scale (which is taken to be the $Z$-boson mass $M_{Z}$ ) to the scale $\mu$. The running is governed by:

$$
\begin{aligned}
\frac{8 \pi^{2}}{g_{i}^{2}(\mu)} & =\frac{8 \pi^{2}}{g_{i}^{2}\left(M_{Z}\right)}+b_{i} \log \left(\frac{\mu}{M_{Z}}\right), & i & =1,2,3 \\
b_{1} & =-\left(80 n_{f}+6 n_{H}\right), & b_{2} & =\frac{22}{3}-\frac{4}{3} n_{f}-\frac{n_{H}}{6}, \quad b_{3}=11-\frac{4}{3} n_{f},
\end{aligned}
$$

for $n_{f}$ families of particles and $n_{H}$ Higgs doublets. Notice that while $b_{2}$ and $b_{3}$ are standard (see, e.g., [36]), the $\mathrm{U}(1)$ coupling constant $g_{1}$ as well as its $\beta$-function are related to the "standard" hypercharge via $g_{1}=g_{Y} / 6$. We also use $g_{3}^{2}\left(M_{Z}\right) / 4 \pi \cong 0.118, g_{E M}^{2}\left(M_{Z}\right) / 4 \pi \cong 1 / 128$, $g_{Y}\left(M_{Z}\right)=g_{E M}\left(M_{Z}\right) / \cos \theta_{W}\left(M_{Z}\right), g_{2}\left(M_{Z}\right)=g_{E M}\left(M_{Z}\right) / \sin \theta_{W}\left(M_{Z}\right)$, and $\sin ^{2} \theta_{W}\left(M_{Z}\right) \cong$ 0.23 , where $\theta_{W}\left(M_{Z}\right)$ is the Weinberg angle. 
Using the SM particle content, setting $n_{f}=3$ and $n_{H}=1$, and making use of (3.19) in (3.18), we solve for the critical energy scale $\mu_{\text {critical }}$ at which the effects of the fractionalcharge instantons surpass the BPST ones. We list the values of $\mu_{\text {critical }}$ in the last column in table 1. Clearly, all values of $\mu_{\text {critical }}$ are super-Planckian for the gauging of $\mathbb{Z}_{6}^{(1)}$ or its subgroups. This is attributed to the fact that the hyper-charge coupling constant is extremely small at the weak scale. One can push $\mu_{\text {critical }}$ into sub-Planckian values provided that extra matter charged under $\mathrm{U}(1)$ participates in the running. ${ }^{15}$

Finally, we might be interested in the energy scale at which the fractional instantons become more important than BPST solutions with minimum topological charge $Q_{(2)}=1$. In this case we simply set $|\Delta B|=1$ in the inequality (3.18) to find that $\mu_{\text {critical }}$ are not dramatically bigger than the values in table 1 .

\section{Cosmological bounds and discussion}

The strongest cosmological constraints on the size of a $\mathbb{T}^{3}$ with sides of order $L$ come from the $\mathrm{CMB}$ and indicate that $L>\mathcal{O}($ few $) \times L_{0}$, where $L_{0} \sim 14 \mathrm{Gpc}$ is the radius of the last scattering surface, see e.g. [27-29]. If compactness of the space is a necessary condition to make sense of the fractional-charge instantons, which is the conservative perspective that we have been following throughout our analysis (however, see the discussion below), then these instantons could have played a role in the early Universe provided that there is extra charged matter and the size of $\mathbb{T}^{3}$ was smaller than or equal to the horizon size when the Universe was hot and dense.

To this end, we trace back the size of $\mathbb{T}^{3}$ before the time of last scattering, when the Universe was deep in the radiation-dominated era at temperature $T$ and the instanton effects are expected to be important. We use the Friedmann equation of a single-component Universe $3 M_{P}^{2} H^{2}(t)=\frac{\pi^{2}}{15} n_{*} T^{4}\left(M_{P}\right.$ is the reduced Planck mass, $H(t)$ is the Hubble parameter, and $n_{*}$ is the number of effective degrees of freedom), along with the fact that the scale factor behaves as $a \propto 1 / \sqrt{H(t)}$. Recalling that the horizon size is $L_{H}(t)=H^{-1}(t)$, we can use this information to obtain the horizon size, the physical size $L_{\mathbb{T}^{3}}(T)$ of $\mathbb{T}^{3}$, and the ratio $\frac{L_{\mathbb{T}^{3}}(T)}{L_{H}(T)}$ at temperature $T$ :

$$
L_{H}(T) \sim \frac{M_{P}}{T^{2}}, \quad L_{\mathbb{T}^{3}}(T)=\mathcal{O}(\text { few }) \frac{M_{P}}{T_{l} T}, \quad \frac{L_{\mathbb{T}^{3}}(T)}{L_{H}(T)}=\mathcal{O}(\text { few }) \times \frac{T}{T_{l}},
$$

where $T_{l}$ is the CMB temperature at the time of last scattering. Since we are interested in temperature much higher than $T_{l}$, we immediately see that the $\mathbb{T}^{3}$ was always bigger than the horizon size. One might immediately run into the conclusion that such fractional solutions should be dismissed since their size has always been bigger than the horizon. This, however, is a very conservative view that needs to be carefully examined in a future study. Moreover, it is conceivable that such solutions can be found at a scale cut off by the horizon.

\footnotetext{
${ }^{15}$ It is important to stress that any extra charged matter must respect the modified cocycle conditions (2.11). We also assume that such new particles have mass above the $\mathrm{TeV}$ scale. As an example, we can assume $\sim 60$ extra scalar particles of mass $\sim 10^{5} \mathrm{GeV}$, which are sterile under $\mathrm{SU}(2)$ and $\mathrm{SU}(3)$ but have a charge 6 under $\mathrm{U}(1)$. They push the critical scale down to $\mu_{\text {critical }} \sim 10^{13} \mathrm{GeV}$ for $G_{\mathrm{SM}}^{2}$. Even at this energy, the $\mathrm{U}(1)$ coupling is still in the perturbative regime.
} 
Assuming one can make sense of the fractional instantons on scales smaller than or equal to the horizon size, then things become more interesting at temperature above the electroweak scale. Now, the electroweak symmetry is restored and there is no barrier between the pre-vacua of $\mathrm{SU}(2)$. In this case the baryon and lepton number violation happens in units of 3 , one per family, at a rate set by temperature $\sim T^{4}$. If there is no extra matter charged under $\mathrm{U}(1)$, then our conclusion from the previous section implies that fractional instantons did not play any role during the thermal history of the Universe. If, however, there is extra matter that makes the fractional instantons more important at some critical energy scale $\mu_{\text {critical }}<M_{P}$, then at temperature $T \gtrsim \mu_{\text {critical }}$ the barrier due to the fractional instantons is removed and the baryon and lepton number violation is expected to proceed at similar rates $\sim T^{4}$ in units of $\Delta B>3$, as given in table 1 .

\section{Acknowledgments}

M.A. is supported by STFC through grant ST/T000708/1. EP is supported by a Discovery Grant from NSERC.

Open Access. This article is distributed under the terms of the Creative Commons Attribution License (CC-BY 4.0), which permits any use, distribution and reproduction in any medium, provided the original author(s) and source are credited.

\section{References}

[1] O. Aharony, N. Seiberg and Y. Tachikawa, Reading between the lines of four-dimensional gauge theories, JHEP 08 (2013) 115 [arXiv: 1305.0318] [INSPIRE].

[2] D. Tong, Line Operators in the Standard Model, JHEP 07 (2017) 104 [arXiv:1705. 01853] [INSPIRE].

[3] D. Gaiotto, A. Kapustin, N. Seiberg and B. Willett, Generalized Global Symmetries, JHEP 02 (2015) 172 [arXiv: 1412.5148] [inSPIRE].

[4] D. Gaiotto, A. Kapustin, Z. Komargodski and N. Seiberg, Theta, Time Reversal, and Temperature, JHEP 05 (2017) 091 [arXiv: 1703.00501] [INSPIRE].

[5] D. Gaiotto, Z. Komargodski and N. Seiberg, Time-reversal breaking in $Q C D_{4}$, walls, and dualities in $2+1$ dimensions, JHEP 01 (2018) 110 [arXiv:1708.06806] [INSPIRE].

[6] I. García-Etxebarria and M. Montero, Dai-Freed anomalies in particle physics, JHEP 08 (2019) 003 [arXiv: 1808.00009] [INSPIRE].

[7] J. Wang and X.-G. Wen, Nonperturbative definition of the standard models, Phys. Rev. Res. 2 (2020) 023356 [arXiv: 1809.11171] [INSPIRE].

[8] J. Davighi, B. Gripaios and N. Lohitsiri, Global anomalies in the Standard Model(s) and Beyond, JHEP 07 (2020) 232 [arXiv:1910.11277] [INSPIRE].

[9] Z. Wan and J. Wang, Beyond Standard Models and Grand Unifications: Anomalies, Topological Terms, and Dynamical Constraints via Cobordisms, JHEP 07 (2020) 062 [arXiv: 1910.14668] [INSPIRE]. 
[10] Z. Wan, J. Wang and Y. Zheng, Higher anomalies, higher symmetries, and cobordisms II: Lorentz symmetry extension and enriched bosonic/fermionic quantum gauge theory, Ann. Math. Sci. Appl. 05 (2020) 171 [arXiv:1912.13504] [INSPIRE].

[11] J. Wang, Anomaly and Cobordism Constraints Beyond the Standard Model: Topological Force, arXiv:2006.16996 [INSPIRE].

[12] G. 't Hooft, A Property of Electric and Magnetic Flux in Nonabelian Gauge Theories, Nucl. Phys. B 153 (1979) 141 [INSPIRE].

[13] G. 't Hooft, Aspects of Quark Confinement, Phys. Scripta 24 (1981) 841 [INSPIRE].

[14] P. van Baal, Some Results for SU(N) Gauge Fields on the Hypertorus, Commun. Math. Phys. 85 (1982) 529 [INSPIRE].

[15] A. Gonzalez-Arroyo, Yang-Mills fields on the four-dimensional torus. Part 1: Classical theory, in Advanced Summer School on Nonperturbative Quantum Field Physics, pp. 57-91 (1997) [hep-th/9807108] [INSPIRE].

[16] P. van Baal, QCD in a finite volume, hep-ph/0008206 [INSPIRE].

[17] M. Ünsal, TQFT at work for IR-renormalons, resurgence and Lefschetz decomposition, arXiv:2106.14971 [INSPIRE].

[18] M. Ünsal, Strongly coupled QFT dynamics via TQFT coupling, JHEP 11 (2021) 134 [arXiv:2007.03880] [INSPIRE].

[19] M. Lachieze-Rey and J.-P. Luminet, Cosmic topology, Phys. Rept. 254 (1995) 135 [gr-qc/9605010] [INSPIRE].

[20] G. 't Hooft, Some Twisted Selfdual Solutions for the Yang-Mills Equations on a Hypertorus, Commun. Math. Phys. 81 (1981) 267 [InSPIRE].

[21] A.A. Belavin, A.M. Polyakov, A.S. Schwartz and Y.S. Tyupkin, Pseudoparticle Solutions of the Yang-Mills Equations, Phys. Lett. B 59 (1975) 85 [InSPIRE].

[22] G. 't Hooft, Computation of the Quantum Effects Due to a Four-Dimensional Pseudoparticle, Phys. Rev. D 14 (1976) 3432 [Erratum ibid. 18 (1978) 2199] [INSPIRE].

[23] G. 't Hooft, Symmetry Breaking Through Bell-Jackiw Anomalies, Phys. Rev. Lett. 37 (1976) 8 [INSPIRE].

[24] M. Garcia Perez, A. Gonzalez-Arroyo and C. Pena, Perturbative construction of selfdual configurations on the torus, JHEP 09 (2000) 033 [hep-th/0007113] [INSPIRE].

[25] A. González-Arroyo, Constructing SU(N) fractional instantons, JHEP 02 (2020) 137 [arXiv:1910.12565] [INSPIRE].

[26] P. van Baal, SU(N) Yang-Mills Solutions With Constant Field Strength on $T^{4}$, Commun. Math. Phys. 94 (1984) 397 [inSPIRE].

[27] J.J. Levin, E. Scannapieco and J. Silk, Is the universe infinite or is it just really big?, Phys. Rev. D 58 (1998) 103516 [astro-ph/9802021] [INSPIRE].

[28] J.R. Bond, D. Pogosian and T. Souradeep, Computing CMB anisotropy in compact hyperbolic spaces, Class. Quant. Grav. 15 (1998) 2671 [astro-ph/9804041] [INSPIRE].

[29] G. Aslanyan, A.V. Manohar and A.P.S. Yadav, The Topology and Size of the Universe from CMB Temperature and Polarization Data, JCAP 08 (2013) 009 [arXiv: 1304.1811] [InSPIRE]. 
[30] A. Kapustin and N. Seiberg, Coupling a QFT to a TQFT and Duality, JHEP 04 (2014) 001 [arXiv:1401.0740] [INSPIRE].

[31] J. Greensite, An introduction to the confinement problem, Lect. Notes Phys. 821 (2011) 1 [INSPIRE].

[32] A.A. Cox, E. Poppitz and F.D. Wandler, The mixed 0-form/1-form anomaly in Hilbert space: pouring the new wine into old bottles, JHEP 10 (2021) 069 [arXiv:2106.11442] [INSPIRE].

[33] K.-M. Lee and P. Yi, Monopoles and instantons on partially compactified D-branes, Phys. Rev. D 56 (1997) 3711 [hep-th/9702107] [INSPIRE].

[34] T.C. Kraan and P. van Baal, Periodic instantons with nontrivial holonomy, Nucl. Phys. B 533 (1998) 627 [hep-th/9805168] [INSPIRE].

[35] P.J. Braam and P. van Baal, Nahm's Transformation for Instantons, Commun. Math. Phys. 122 (1989) 267 [INSPIRE].

[36] S.P. Martin, A Supersymmetry primer, Adv. Ser. Direct. High Energy Phys. 18 (1998) 1 [hep-ph/9709356] [INSPIRE]. 